\title{
Parámetros biológicos de Tetranychus desertorum (Acari: Tetranychidae) sobre hojas de poroto
}

\author{
Biological parameters of Tetranychus desertorum \\ (Acari: Tetranychidae) on bean leaves
}

Víctor Tello M. ${ }^{1}$, Pamela Castillo M. ${ }^{1}$, Romina Briceño V. ${ }^{1}$, Matías Sánchez M. ${ }^{1}$

\section{RESUMEN}

Se estudió la biología de Tetranychus desertorum sobre Phaseolus vulgaris Linnaeus var. Canario bajo condiciones controladas de $25,0 \pm 2,0^{\circ} \mathrm{C}$ de temperatura, 70,0 $\pm 5,0 \% \mathrm{HR}$ y un fotoperíodo de $12: 20 \mathrm{~h}(\mathrm{~L}: \mathrm{O})$. El ciclo de huevo a adulto duró $11,3 \pm 0,1$ días, con una supervivencia del $62,8 \%$. La proporción sexual fue de $83,7 \%$ hembras: $23,7 \%$ machos. La longevidad media de las hembras fue de 15,5 $\pm 0,8$ días y la tasa de oviposición diaria fue de 4,8 $\pm 0,2$ huevos/hembra/día. La tasa intrínseca de incremento $\left(r_{m}\right)$ fue de 0,188 ; la tasa finita de crecimiento $(\lambda)$ fue de 1,207 individuos/hembra/día; el tiempo generacional medio $(T)$ fue de 18,24 días y la tasa neta de reproducción $\left(R_{0}\right)$ fue de 30,85. Los patrones de desarrollo de T. desertorum están dentro de los descritos para ácaros de la familia Tetranychidae.

Palabras clave: desarrollo posembrionario, fecundidad, supervivencia, tabla de vida.

\begin{abstract}
The study on the biology of Tetranychus desertorum on Phaseolus vulgaris Linnaeus var. canario was carried out under controlled conditions of $25.0 \pm 2.0^{\circ} \mathrm{C}$ temperature, $70.0 \pm 5.0 \% \mathrm{RH}$ and $12: 12 \mathrm{~h}(\mathrm{~L}: \mathrm{D})$ photophase. The egg to adult time span lasted $11.33 \pm 0.11$ days, with a $62.8 \%$ survival. The sexual ratio was $83.7 \%$ females: 23.7 males. The mean female longevity was $15.5 \pm 0.8$ days and the daily mean oviposition was $4.8 \pm 0.2$ eggs/female/day. The intrinsic rate of increase $\left(r_{m}\right)$ was $0.188 ;$ the finite rate of increase ( $\lambda)$ was of 1.207 individuals/female/day; the mean time span of one generation $(T)$ was 18.24 days; and the net rate of reproduction $\left(R_{0}\right)$ was 30.85. The development patterns of $\mathrm{T}$. desertorum fall within those described for mites of the family Tetranychidae.
\end{abstract}

Key words: postembryonic development, fecundity, survival, life table.

\section{Introducción}

Los ácaros (Orden Acarina) de la familia Tetranychidae están entre las plagas más importantes de plantas cultivadas. La familia Tetranychidae incluye un número de especies de importancia económica, entre las cuales Tetranychus urticae Koch, 1836, la arañita carmín T. cinnabarinus (Boisduval, 1867) Boudraux y Tetranychus desertorum Banks, 1900; son importantes. La arañita roja desertícola se encuentra asociada a más de 173 plantas hospederas, distribuyéndose en Argentina, Brasil, China, Estados Unidos, Hawai, México y Venezuela, entre otros países (Bolland et al. 1998). En Chile se asocia principalmente a poroto (Phaseolus vulgaris
Linnaeus, 1753), alfalfa (Medicago sativa Linnaeus, 1753), alcayota (Cucurbita ficifolia Bouché, 1837), sandía (Citrullus lanatus (Thunberg) [Matsumura \& Nakai, 1916]) y damasco (Prunus armeniaca Linnaeus, 1753) (Klein y Waterhouse, 2000).

La reproducción en los tetraníquidos es muy sensible a una amplia variedad de condiciones intrínsecas y extrínsecas. Los factores intrínsecos que influencian los parámetros de tabla de vida de estos ácaros incluyen la raza y el nivel de endogamia, densidad de la colonia, edad de las hembras y de la población, y fertilidad de las hembras (Wrensch 1985). Los factores extrínsecos incluyen la temperatura, humedad, luz, depredación, y también diferentes características de las plantas hospederas,

\footnotetext{
$1 \quad$ Facultad de Recursos Naturales Renovables, Universidad Arturo Prat. Avenida Arturo Prat 2120, Casilla 121, Iquique, Chile. Autor por correspondencia: vtello@unap.cl.
} 
como la variedad, estado nutricional y edad de la planta (Adango et al., 2006; Vásquez et al., 2007).

En la zona norte de Chile $T$. desertorum es una plaga primaria, distribuida, e importante para el cultivo del poroto, pero no existe información sobre la biología ni de los daños producidos por este ácaro. El objetivo de este estudio fue determinar los parámetros de tabla de vida de la arañita desertícola T. desertorum sobre hojas de poroto, con el propósito de sentar las bases para futuras investigaciones que apunten a determinar el impacto de este ácaro en la producción de esta leguminosa y desarrollar programas de manejo integrado.

\section{Materiales y Métodos}

Los bioensayos se hicieron en los laboratorios del Departamento de Agricultura del Desierto y Biotecnología de la Universidad Arturo Prat (Iquique-Chile), a $26,0 \pm 1,0{ }^{\circ} \mathrm{C}, 65,0 \pm 5,0 \% \mathrm{HR}$ y un fotoperíodo de 12:12 h (L:O) entre el 2 de junio al 14 de septiembre del 2009. T. desertorum se colectó de plantas de alfalfa en la Estación Experimental Canchones, ubicada en la Pampa del Tamarugal (lat $32^{\circ} 49^{\prime} \mathrm{S}$; long $71^{\circ} 17^{\prime} \mathrm{W}$ ), dependiente del Departamento de Agricultura del Desierto y Biotecnología. Se multiplicaron en plantas de poroto, Phaseolus vulgaris L. var. Canario, en secuencias que se infestaron cada siete días. Las plantas provinieron de la Estación Experimental Canchones.

\section{Desarrollo posembrionario}

Para determinar los parámetros poblacionales de $T$. desertorum se separaron hojas de poroto de madurez y tamaño estándar, que se pusieron con la superficie abaxial hacia arriba sobre una capa de algodón saturado de agua en placas Petri plásticas de $12 \mathrm{~cm}$ de diámetro. Estas placas Petri, a su vez, se pusieron en bandejas plásticas de $41 \times 31 \times 7 \mathrm{~cm}$ con una esponja de poliuretano saturada de agua. En cada hoja se dispuso una hembra grávida para obtener huevos. Estos sustratos se revisaron después de $24 \mathrm{~h}$ para verificar la ovipostura. Se dejó un huevo/disco, y se removió el exceso de huevos y también la hembra. Las evaluaciones del tiempo de desarrollo de cada estadio, desde huevos a adultos de machos y hembras, se hicieron dos veces al día (9:00 y 16:00). Cada unidad experimental (un huevo/disco) se repitió 13 veces. Los resultados de desarrollo posembrionario se transformaron por $\log (\mathrm{x}+1)$ (Zar 2006). Posteriormente se aplicó un ANDEVA y la prueba de comparación múltiple de Tukey (Zar 2006).

\section{Preoviposición, oviposición, posoviposición y longevidad}

Estos períodos adultos fueron estudiados en un grupo de 13 hembras de menos de 24 horas de edad. Cada hembra se dispuso junto a un macho en una hoja de poroto aislada siendo observada a intervalos de 12 horas. Para obtener la proporción sexual, los huevos puestos por cada hembra se registraron diariamente, se retiraron de la hoja y se ubicaron en otras placas hasta la emergencia de los adultos. Los períodos de oviposición y posoviposición de las hembras fueron calculados desde el momento en que el primer huevo fue depositado hasta la postura del último huevo y desde el momento en que el último huevo fue puesto hasta la muerte de la hembra, respectivamente.

\section{Tabla de vida}

El cálculo de la tabla de vida de T. desertorum se construyó siguiendo la metodología propuesta por Birch (1948). La tasa de supervivencia $\left(l_{x}\right)$ fue expresada como el número de individuos vivos a un tiempo " $x$ ", mientras que la fecundidad a una edad específica $\left(m_{x}\right)$ se calculó tomando como base el número de hembras descendientes producidas a partir de una hembra en un tiempo $x$. Los parámetros poblacionales estimados fueron: tasa neta de reproducción $\left(R_{0}\right)$, tiempo generacional $(T)$, tasa intrínseca de crecimiento $\left(r_{m}\right)$, y tasa finita de crecimiento natural $(\lambda)$, que se estimaron a partir de los resultados de fecundidad y supervivencia de las hembras. Los parámetros de la tabla de vida se calcularon con el programa computacional LIFE 48 escrito en BASIC (Abou-Setta et al., 1986).

\section{Resultados y Discusión}

\section{Desarrollo posembrionario}

En la Tabla 1 se presentan los estadísticos para el tiempo de duración de los diferentes estados de desarrollo de la arañita desertícola. El período de cada estadio presentó diferencias altamente significativas entre ellos $\left(F_{3 ; 84}=36,40 ; P<0,0001\right)$, con la 
Tabla 1. Duración promedio, en días, de los estados posembrionarios de T. desertorum $(\mathrm{n}=13)$ desarrollados sobre poroto, Phaseolus vulgaris L. var. Canario.

\begin{tabular}{lllccr}
\hline \multirow{2}{*}{ Parámetros estadísticos } & \multicolumn{5}{c}{ Duración en días } \\
\cline { 2 - 6 } & Huevos & Larvas & Protoninfas & Deutoninfas & Hembras adultas \\
\hline Media $^{1}$ & $4,86 \mathrm{a}$ & $2,14 \mathrm{ab}$ & $1,91 \mathrm{c}$ & $2,73 \mathrm{~b}$ & 11,33 \\
E.E $^{2}$ & 0,20 & 0,18 & 0,17 & 0,19 & 0,11 \\
\hline
\end{tabular}

1 Medias con distinta letra minúscula en la fila son estadísticamente diferentes según prueba de Tukey $(P<0,05)$.

2 EE: error estándar.

mayor duración para la incubación de los huevos, la que varió entre tres y seis días, y los de menor duración fueron los estados de larva y protoninfa, que variaron entre uno y cuatro días para ambos estadios. El desarrollo completo hasta la hembra adulta varió entre 11 y 12 días.

Los resultados para el desarrollo de $T$. desertorum están dentro de los patrones de variación de ácaros Tetranychidae. Rivero y Vásquez (2009) obtuvieron un período de incubación de 3,8 días para $T$. desertorum sobre poroto, siendo este estadio el de mayor duración. Carey y Bradley (1982) encontraron períodos de incubación para tres especies de tetraníquidos variando entre 4,4 y 4,9 días a $23,8^{\circ} \mathrm{C}$. Crooker (1985) indicó que el período de desarrollo desde huevo hasta adulto en los Tetranychidae puede variar desde seis a 10 días o más, dependiendo de las especies, temperatura, humedad, planta hospedera y otros factores. Similar a lo registrado por Biswas et al. (2004), la baja humedad relativa no afectó a ninguno de los estados posembrionarios. De acuerdo con Jeppson et al. (1975), al estudiar los parámetros de vida de T. desertorum, determinaron que los períodos de incubación variaron entre dos y cinco días; estos mismos autores obtuvieron para Tetranychus evansi Baker \& Pritchard 1960 un período de incubación de tres días a $23{ }^{\circ} \mathrm{C}$ y $50 \%$ HR.

La duración de la larva, protoninfas y deutoninfas de nuestros resultados son similares a los registrados por Rivero y Vásquez (2009) para $T$. desertorum criada sobre poroto, quienes obtuvieron una duración promedio de 1,$4 ; 1,0$ y 0,7 días para larva, protoninfa y deutoninfa, respectivamente. Kazak y Kibritçi (2008) para $T$. cinnabarinus criada sobre ocho cultivares de fresa (Fragaria $\times a n a n a s s a$ Duchesne, 1758) quienes obtuvieron rangos entre 2,0 y 2,$2 ; 1,6$ y 1,9 y 2,1 y 2,3 días, respectivamente, a $25^{\circ} \mathrm{C}, 65 \%$ de $\mathrm{HR}$ y
14:10 L:O. Para Oligonychus perseae Tuttle, Baker \& Abbatiello 1976, desarrollado sobre hojas de aguacate, Persea americana Millar 1768 , a $30^{\circ} \mathrm{C}$; 60-80\% HR y 12 horas de luz, Ramírez et al. (1993) registraron 1,7 días para la etapa larval; 1,2 días para las protoninfas y 1 , días para las deutoninfas. Morros y Aponte (1994) obtuvieron una duración de 1,8 1,3 y 1,9 días para larvas, protoninfas y deutoninfas, respectivamente, para Tetranychus ludeni Zacher, 1913, desarrollado sobre hojas aisladas de poroto a $26,3 \pm 3,9^{\circ} \mathrm{C}$ y $69,4 \pm 19,5 \%$ de HR. Gotoh y Gomi (2003) estudiaron el desarrollo de dos especies de tetraníquidos muy relacionadas, Tetranychus kanzawai Kishida, 1927, y T. parakanzawai Ehara, 1999 , a $25^{\circ} \mathrm{C}, 16: 8 \mathrm{~h}$ (L:O). La duración de cada estadio fue, para $T$. kanzawai, entre 1,0 a 1,9 días para las larvas; 0,6 a 1,6 días para las protoninfas y 0,3 a 1,7 días para las deutoninfas. En el caso de T. parakanzawai obtuvieron una duración de un día para las larvas; entre 0,8 a 0,9 días para las protoninfas y entre 0,4 a 0,9 días para las deutoninfas.

Biswas et al. (2004) registraron para T. cinnabarinus sobre Solanum melongena L., 1753, una duración de 1,5 días para el período larval, 1,3 días para las protoninfas y 1,9 días para las deutoninfas entre $21-31{ }^{\circ} \mathrm{C}$. Adango et al. (2006) obtuvieron para T. ludeni, alimentado con Amaranthus cruentus L., 1759, y Solanum macrocarpon Linnaeus 1753, una duración para las larvas de 1,9 y 2,0 días; para las protoninfas de 1,6 y 1,8 días y para las deutoninfas de 1,9 y 2,1 días, respectivamente, a $27^{\circ} \mathrm{C}, 70 \%$ HR y $12: 12 \mathrm{~h}$ (L:O). Noronha (2006) indicó que Tetranychus marianae McGregor, 1950, alimentado sobre Passiflora edulis Sims 1818 , F. flavicarpa Deg. a una temperatura de $25 \pm 1{ }^{\circ} \mathrm{C}, 80 \pm 10 \%$ HR y $12: 12$ h (L:O) presentó una duración de 1,4 días para las larvas, 0,9 días para las protoninfas y 1,3 días para las deutoninfas. 


\section{Fases adultas}

Tanto las fases adultas (preoviposición, oviposición, posoviposición, además de la longevidad) como las tasas de oviposición (Tabla 2) son coincidentes con lo registrado por la literatura para especies de Tetranychidae. Rivero y Vásquez (2009) obtuvieron una duración promedio de 1,1; 8,4 y 1,3 días para los períodos de preoviposición, oviposición y posoviposición, respectivamente, para T. desertorum en poroto. Estos autores registraron una fertilidad de 6,9 huevos/hembra/día al cuarto día desde el comienzo de la ovipostura y una longevidad, para las hembras, de 10 días. Kazak y Kibritçi (2008), obtuvieron rangos entre 1,2 y 1,5; 13,2 y 20,$1 ; 2,5$ y 4,5 y 18,0 y 25,3 días para los períodos de preoviposición, oviposición, posoviposición y longevidad, respectivamente, para T. cinnabarinus sobre fresa. Estos mismos autores encontraron una oviposición por hembra entre 62,7 y 163,4 huevos y una tasa de oviposición diaria entre 4,8 y 8,5 huevos a $25 \pm 2{ }^{\circ} \mathrm{C}, 65 \pm 10 \%$ HR y $14: 10 \mathrm{~h}$ (L:O).

La duración promedio en días para T. ludeni, fue de 1,$2 ; 14,7$ y 2,4 días para los períodos de preoviposición, oviposición y posoviposición, respectivamente. La fertilidad máxima fue de 118,1 huevos/hembra y tasa de oviposición fue de 7,9 huevos/hembra/día. La longevidad obtenida para esta especie a $26,3 \pm 3,9{ }^{\circ} \mathrm{C}$ y $69,4 \pm 19,5 \% \mathrm{HR}$ fue de 18,4 días (Morros y Aponte 1994). Adango et al. (2006) obtuvieron para T. ludeni una duración para los períodos de preoviposición, oviposición, posoviposición y longevidad (hembras) de 1,3; 15,6; 1,9 y 28,3 días sobre $A$. cruentus y de 1,$2 ; 12,9 ; 0,8$ y 24,9 días sobre $S$. macrocarpon.

Tabla 2. Duración promedio, en días, de los períodos biológicos del estado de hembra adulta y tasa de oviposición media diaria de T. desertorum $(\mathrm{n}=13)$ sobre frejol Phaseolus vulgaris L. var Canario.

\begin{tabular}{lr}
\hline Períodos & Duración promedio (días) $\pm \mathrm{EE}^{1}$ \\
\hline Preoviposición & $2,15 \pm 0,22$ \\
Oviposición & $12,23 \pm 0,69$ \\
Posoviposición & $1,08 \pm 0,40$ \\
Longevidad & $15,46 \pm 0,76$ \\
Oviposición & Promedio de huevos $\pm \mathrm{EE}^{1}$ \\
Huevos/hembra & $58,69 \pm 4,55$ \\
Huevos/hembra/día & $4,75 \pm 0,19$ \\
\hline
\end{tabular}

1 EE: error estándar.
Biswas et al. (2004) obtuvieron para T. cinnabarinus una duración para el período reproductivo entre 9,1 y 15,3 y longevidad entre 15,0 y 29,9 días sobre S. melongena. La fertilidad máxima varió entre 24,3 y 33,0 huevos/hembra. La fertilidad máxima obtenida sobre $A$. cruentus y $S$. macrocarpon fue de 111,6 $\pm 10,7$ y $84,3 \pm 10,5$ huevos/hembra, respectivamente. Noronha (2006) obtuvo una duración para los períodos de preoviposición y oviposición en T. marianae sobre P. edulis de 1,1 y 19,9 días, respectivamente. La longevidad determinada para las hembras fue de 24,5 días. La fertilidad máxima fue de 75,5 $\pm 6,2$ huevos/hembras y la tasa de ovipostura fue de 3,7 $\pm 0,2$ huevos/hembra/día. Bonato et al. (2000) obtuvieron una tasa de ovipostura (huevos/hembra) y una longevidad (días) para las hembras de Tetranychus ogmophallos Ferreira \& Flechtmann 1997, de 60,0 $\pm 2,9$ y 16,5 $\pm 0,8$ sobre Arachis hypogaea Linnaeus, 1753; 63,9 $\pm 5,7$ y $15,4 \pm 1,2$ sobre Glycine max (Linnaeus) Merrill, 1917 y $104,3 \pm 7,8$ y $25,3 \pm 1,1$ sobre $P$. vulgaris, respectivamente.

\section{Parámetros de tabla de vida}

La supervivencia de los estados juveniles fue de $62,8 \%(\mathrm{n}=156)$ y la proporción o razón sexual fue de $83,7 \%$ de hembras $(n=156)$. Nuestros resultados concuerdan con los registrados por Bonato et al. (2000), con $85,0 \pm 5,0 \%$ de supervivencia para juveniles de T. ogmophallos y con los obtenidos, por esos mismos autores, para A. cruentus y $S$. macrocapon con porcentajes de supervivencia de 45,0 y $72 \%$, respectivamente. La supervivencia de los juveniles, además de los factores abióticos, puede ser influenciada por la calidad del hospedero (Kerguelen y Hoddle 2000). De esta manera, T. cinnabarinus pudo haber encontrado en esta variedad de clavel los nutrientes adecuados que favorecieron su reproducción, así lo confirman los estudios de Kazan y Kibritçi (2008) con este tetraníquido en diferentes cultivares de fresa.

Respecto de la proporción sexual, nuestros resultados coinciden con lo observado por Noronha (2006) para T. marianae con $81,0 \%$ de hembras y con los registrados por Adango et al. (2006) para T. ludeni desarrollado sobre A. cruentus y $S$. macrocarpon con 72,72 y $78,68 \%$ de hembras, respectivamente. De forma similar, Bonato et al. (2000) observaron una proporción sexual de $80 \%$ hembras para T. ogmophallos sobre tres especies de fabáceas (A. hypogaea, G. max 
y P. vulgaris). Contrariamente a nuestros resultados, Morros y Aponte (1994) registraron para T. ludeni una proporción sexual de 62,4\% de hembras. De acuerdo con Boudreaux (1963), esta variación se debe a que no existe una distribución normal de la proporción sexual en los tetraníquidos y por lo tanto la relación de sexos depende de la cantidad de espermatozoides introducidos durante la cópula y del tiempo de duración de la misma.

La tasa de oviposición diaria máxima se alcanzó al quinto día desde el comienzo de la ovipostura y se mantuvo a niveles altos hasta al día 10, a partir del cual la tasa comenzó a decaer. La tasa de supervivencia fue máxima (100\%) hasta el día 10 , a partir del cual comenzó a disminuir, aunque manteniéndose hasta el día 14 sobre el $80 \%$, alcanzando el 50\% de mortalidad el día 16 (Figura 1). La tasa neta de reproducción $\left(R_{0}\right)$ y la tasa intrínseca de crecimiento $\left(r_{m}\right)$ son indicadores importantes de las dinámicas poblacionales de los tetraníquidos (Sabelis, 1985; Krisp et al., 1998). Las comparaciones de $R_{0}$ y $r_{m}$ ofrecen mayor información que la disponible de análisis independientes de parámetros de vida individuales (Zhang et al. 2007).

Para la tabla de vida de fecundidad (Tabla 3), la tasa neta de reproducción $\left(R_{0}\right)$ fue 30,850 y el

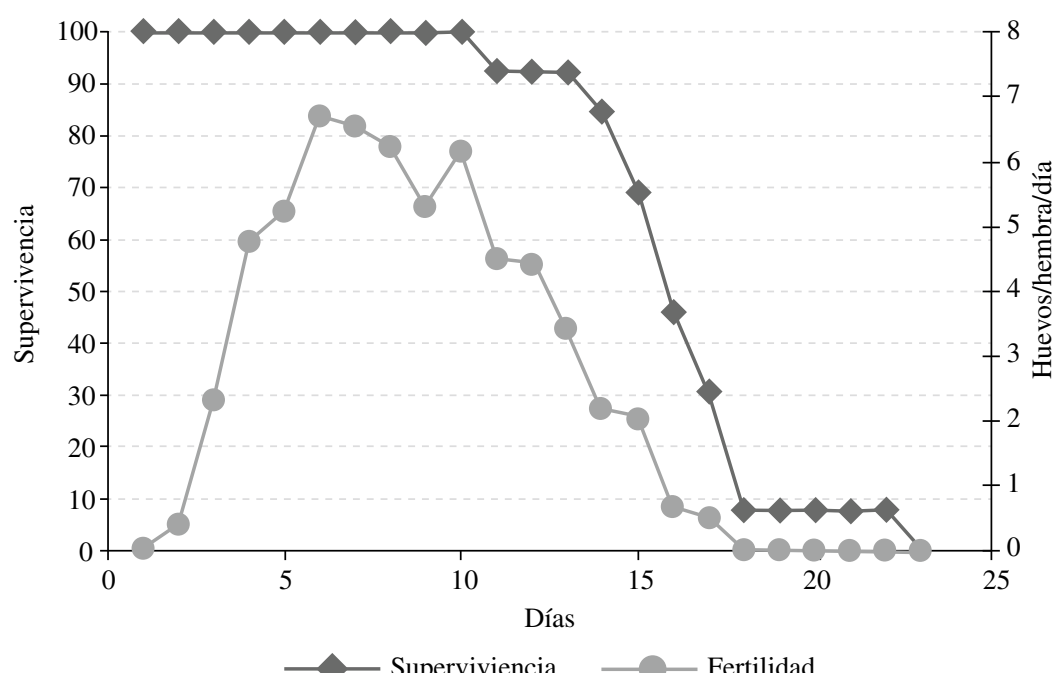

Figura 1. Tasa de supervivencia y fertilidad a una edad específica de Tetranychus desertorum sobre poroto, Phaseolus vulgaris L. var Canario.

Cuadro 3. Parámetros de tabla de vida para especies de arañitas fitófagas del género Tetranychus criados sobre diferentes hospederos, en condiciones experimentales indicadas para cada especie.

\begin{tabular}{|c|c|c|c|c|c|c|}
\hline Tetranychus $^{1}$ & Hospedero & $r_{m}$ & $\lambda$ & $\mathrm{T}$ & $\mathrm{R}_{\mathrm{o}}$ & Referencias \\
\hline T. parakanzawai $(t 1, f 4)$ & Pueraria lobata (Willd.) Ohwi & 0,276 & 1,318 & 22,17 & 134,80 & Gotoh y Gomi (2003) \\
\hline T. cinnabarinus $(t 1, h r 3, f 3)$ & Fragaria $x$ ananassa Duchesne & 0,253 & - & 22,00 & 120,19 & Kazak y Kibritci (2008) \\
\hline T. ludeni $(t 2, h r 3, f 1)$ & Phaseolus vulgaris $L$. & 0,253 & 1,287 & 19,36 & 77,42 & Morros y Aponte (1994) \\
\hline T. ogmophallos $(t 2, h r 4, f 2)$ & Phaseolus vulgaris $L$. & 0,232 & - & 20,70 & 75,90 & Bonato et al. (2000) \\
\hline T. kanzawai $(t 1, f 4)$ & Pyrus pyrifolia (Burm.) & 0,229 & 1,258 & 21,25 & 71,24 & Gotoh y Gomi (2003) \\
\hline T. ludeni $(t 2, h r 3, f 1)$ & Amaranthus cruentus & 0,215 & 1,240 & 17,36 & 41,80 & Adango et al. (2006) \\
\hline T. cinnabarinus $(t 2, h r l, f 3)$ & Dianthus caryophyllus $L$. & 0,200 & 1,222 & 18,55 & 40,96 & Tello et al. (2009) \\
\hline T. desertorum $(t 2, h r 3, f 1)$ & Phaseolus vulgaris $L$. & $\mathbf{0 , 1 8 8}$ & 1,207 & 18,24 & 30,85 & Presente trabajo \\
\hline T. ludeni $(t 2, h r 3, f 1)$ & Solanum macrocarpon $L$. & 0,173 & 1,190 & 16,20 & 16,32 & Adango et al. (2006) \\
\hline T. marianae $(t 1, h r 4, f 1)$ & Passiflora edulis Sims. & 0,172 & 1,187 & 22,81 & 50,14 & Noronha (2006) \\
\hline T. desertorum $(t 2, h r 3, f 1)$ & Phaseolus vulgaris $L$. & 0,144 & 1,155 & 11,15 & 41,10 & Rivero y Vásquez (2009) \\
\hline
\end{tabular}

1 Condiciones de temperatura (t), HR (hr) y fotoperíodo $(f)$ de los ensayos: $t 1=20-25{ }^{\circ} \mathrm{C} ; t 2=26-30{ }^{\circ} \mathrm{C} ; h r 1=40-50 \%$; $h r 2=51$ $60 \% ; h r 3=61-70 \% ; h 4=71-90 \% ; f 1=12 \mathrm{~L}: 12 \mathrm{O} ; f 2=13: 11 ; f 3=14: 10 ; f 4=16: 8$. 
tiempo generacional ( $T$ ) 18,244 días, lo que indica que la arañita tiene la capacidad de aumentar su población 30,850 veces en aproximadamente 18,244 días; es decir, por cada individuo presente en una generación, habrán 30,850 en la generación siguiente. La tasa intrínseca de crecimiento $\left(r_{m}\right)$ fue 0,188 por individuo/día, lo que significa que en cualquier instante el número de individuos de la población de $T$. desertorum se espera que aumente aproximadamente $18,8 \%$ de un día a otro. Convirtiendo este valor a una tasa finita de crecimiento $(\lambda)$ por individuo/hembra/día se obtiene 1,207 ; lo que significa que por cada arañita presente en un día, habría casi 1,21 arañitas al día siguiente.

Los parámetros de la tabla de vida no fueron iguales a los obtenidos por Rivero y Vásquez (2009) para esta misma especie de tetraníquido sobre hojas de poroto. Estos autores obtuvieron una tasa intrínseca de crecimiento de 0,144 ; una tasa neta de reproducción de 41,10 , un tiempo generacional de 11,15 y una tasa finita de crecimiento de 1,155. Estas diferencias podrían ser explicadas por la variedad de poroto utilizada como alimento, que en el caso de ellos se trató del cultivar "Tacarigua". Kazan y Kibritçi (2008) obtuvieron para T. cinnabarinus, sobre diferentes variedades de fresa, tasas intrínsecas de crecimiento entre 0,208 y 0,253 , tasas netas de reproducción entre 39,51 y 120,19 y tiempos generacionales entre 18,96 y 22,32 días. Las diferencias de nuestros resultados con los anteriormente descritos para Kazan y Kibritçi (2008) podrían deberse a los diferentes sustratos vegetales y condiciones experimentales utilizados. Los valores de $r_{m}$ de los tetraníquidos puede ser mayor cuando el tiempo generacional es breve y la fertilidad es alta, puede también ser influenciada por la naturaleza de la planta hospedera, superficie disponible para cada individuo y los valores de temperatura y humedad (Rivero y Vásquez, 2009). En general, los resultados para el desarrollo de T. cinnabarinus están dentro de los rangos de variación para arañitas del género Tetranychus indicados en la literatura.

Los resultados obtenidos en este estudio son la primera información sobre aspectos biológicos de la arañita roja del desierto en Chile y servirán de base para futuras investigaciones para determinar el daño y estrategias de control de este ácaro. Nuevos estudios serán necesarios para determinar su importancia como plaga en otros hospederos y su impacto sobre la producción en esos cultivos.

\section{Literatura Citada}

About-Setta, M.M.; Dorrell, R.R.; Childress, C.C.

1986. Life 48: a BASIC computer program to calculate life table parameters for an insect or mites species. Florida Entomologist 69: 690-697.

Adango, E.; Onzo, A.; Hanna, R.; Atachi, P.; James, B.

2006. Comparative demography of the spider mite, Tetranychus ludeni, on two host plants in West Africa. Journal of Insect Science 6: 49 .

Birch, L.C.

1948. The intrinsic rate of natural increase of an insect population. Journal of Animal Ecology 17: 15-26.

Biswas, G.C.; Islam, W.; Haque, M.M.; Saha, R.K.; Hoque,

K.M.F.; Islam M.S.; Haque, M.E.

2004. Some biological aspects of Carmine Mite, Tetranychus cinnabarinus Boisd. (Acari: Tetranychidae) infesting egg-plant from Rajshahi. Journal of Biological Sciences 4 (5): 588-591.

Bolland, H.R.; Gutiérrez, J.; Fletchmann, C.H.W. 1998. World catalogue of the spider mite family (Acari: Tetranychidae): Leiden, Koninklijke Brill NV, 408 p.

Bonato, O.; Santarosa, L.; Ribeiro, G.; Lucchini, F. 2000. Suitability of three legumes for development of Tetranychus ogmophallos (Acari: Tetranychidae). Florida Entomologist 83 (2): 203-205.

Boudreaux, B.H.

1963. Biological aspects of some phytophagous mites. Annual Review of Entomology 8: 137-154.
Carey, R.J.; Bradley, J.W.

1982. Developmental rates, vital schedules, sex ratios, and life tables for Tetranychus urticae, T. turkestani and $T$. pacificus (Acarina: Tetranychidae) on cotton. Acarologia 23 (4): 333-345.

Crooker, A.

1985. Embryonic and juvenile development - The Tetranychidae. En: Helle, W.; Sabelis, M.W. (eds.) Spider mites: their biology, natural enemies and control. World Crop Pests, Vol 1A. Elsevier, Amsterdam, pp. 149-163.

Gotoh, T.; Gomi, K.

2003. Life-history traits of the Kanzawa spider mite Tetranychus kanzawai (Acari: Tetranychidae). Applied Entomology and Zoology 38 (1): 7-14.

Jeppson, I.R.; Keifer, H.H.; Baker, E.W.

1975. Mites injurious to economic plants. University of California Press. Berkeley. 614 p.

Kazak, C.; Kibritçi, C.

2008. Population Parameters of Tetranychus cinnabarinus Boisduval (Prostigmata: Tetranychidae) on Eight Strawberry Cultivars. Turkish Journal of Agriculture and Forestry 32: 19-27.

Kerguelen, V.; Hoddle, M.S.

2000. Comparison of the susceptibility of several cultivars of avocado to the persea mite, Oligonychus perseae (Acari: Tetranychidae). Scientia Horticulturae 84: 101-114. 
Klein, C.; Waterhouse, D.F.

2000. The distribution and importance of arthropods associated with agriculture and forestry in Chile (Distribución e importancia de los artrópodos asociados a la agricultura y silvicultura en Chile). ACIAR Monograph $\mathrm{N}^{\circ} 68$, Canberra, Australia. 234 p.

Krisp, O.E; Witul, A.; Willems, P.E.L.; Dicke, M.

1998. Intrinsic rate of population increase of the spider mite Tetranychus urticae on the ornamental crop gerbera: intraspecific variation in host plant and herbivore. Entomologia Experimentalis et Applicata 89: 159-168.

Morros, M.; Aponte, O.

1994. Biología y tabla de vida de Tetranychus ludeni Zacher en carota Phaseolus vulgaris L. Agronomía Tropical 44 (4): 667-677.

Noronha, A.

2006. Biological aspects of Tetranychus marianae McGregor (Acari: Tetranychidae) reared on yellow passion fruit (Passiflora edulis Sims f. flavicarpa Deg.) leaves. Revista Brasileira de Zoologia 23 (2): 404-407.

Ramírez, M.; Otero, G.; López, J.; Resendiz, B.

1993. Constantes térmicas y tablas de vida de Oligonychus perseae (Trombidiformes: Tetranychidae). Agrociencia 4 (1): 67-80.

Rivero, E.; Vásquez, C.

2009. Biologia e table de vida de Tetranychus desertorum (Acari: Tetranychidae) sobre folhas de feijão (Phaseolus vulgaris). Zoologia 26 (1): 38-42.
Sabelis, M.W.

1985. Reproductive strategies. En: Helle, W.; Sabelis, M.W. (eds.) Spider mites: their biology, natural enemies and control. World Crop Pests, Vol. 1A. Elsevier, Amsterdam, pp. 265-278.

Tello, V.; Vargas, R.; Araya, J.

2009. Parámetros de vida de Tetranychus cinnabarinus (Acari: Tetranychidae) sobre hojas de clavel, Dianthus caryophyllus. Revista Colombiana de Entomología 35 (1): 47-51.

Vásquez, C.; Colmenarez, M.; Varela, N.; Díaz, L.

2007. Antibiosis of kidney beans cultivars to the carmine spider mite, Tetranychus cinnabarinus (Boisduval) (Acari: Tetranychidae). Integrated Control of Plant-feeding Mites. IOBC/wprs Bulletin 30 (5): 133-138.

Wrensch, D.L.

1985. Reproductive parameters. En: Helle, W.; Sabelis, M.W. (eds.) Spider mites: their biology, natural enemies and control. Amsterdam, Elsevier Science Publishers B.V., vol. 1A, pp. 165-168.

Zar, J.H.

2006. Biostatistical analysis. Prentice-Hall Inc. 5 edition, Englewood Cliffs, New Jersey, USA. 960 p.

Zhang, Z.J.; Wu, Q.J.; Li, X.F.; Zhang, Y.J.; Xu, B.Y.; Zhu, G.R. 2007. Life history of western flower thrips, Frankliniella occidentalis (Thysan., Thripidae), on five different vegetable leaves. Journal of Applied Entomology 131: 347-354. 
